\title{
Generalized Code Index Modulation and Spatial Modulation for High Rate and Energy-Efficient MIMO Systems on Rayleigh Block-Fading Channel
}

\author{
Fatih Cogen, Member, IEEE, Erdogan Aydin ${ }^{\circledR}$, Member, IEEE, Nihat Kabaoglu ${ }^{\circledR}$, Member, IEEE, \\ Ertugrul Basar ${ }^{\circledR}$, Senior Member, IEEE, and Haci Ilhan, Senior Member, IEEE
}

\begin{abstract}
In this article, a high data rate and energy-efficient multiple-input multiple-output transmission scheme is considered by combining two popular and rational modulation techniques: spatial modulation (SM) and code index modulation-spread spectrum (CIM-SS). Since in the considered system, called generalized CIM-SM (GCIM-SM), incoming information bits determine modulated symbols, activated transmit antenna indices as well as their corresponding spreading code indices, data bits are conveyed not only by modulated symbols but also by the indices of the active antenna and spreading codes. Hence, a GCIM-SM scheme accommodates faster data rates while spending less transmission power and possessing better error performance compared to the conventional direct sequence spread spectrum (DS-SS), SM, quadrature SM (QSM), and CIM-SS systems. The mathematical expressions of the GCIM-SM system for bit error rate, throughput, energy efficiency, and the system complexity are derived to analyze the overall system performance. Besides, it has been shown via computer simulations that the GCIM-SM system has lower transmission energy, faster data transmission rate, and better error performance than DS-SS, SM, QSM, and CIM-SS systems. Performance analysis of the considered system was performed on Rayleigh block-fading channels for quadrature amplitude modulation technique.
\end{abstract}

Index Terms-Code index modulation (CIM), direct-sequence spread-spectrum (DS-SS), energy efficiency, index modulation (IM), multiple-input multiple-output (MIMO) systems, quadrature spatial modulation (QSM), spatial modulation (SM).

\section{INTRODUCTION}

$\mathbf{T}$ HE recent developments in technology for wireless communication systems and applications, such as video conference, sharing large files, high-quality video, and audio broadcast applications, gave rise to increased demand for high data

Manuscript received September 24, 2019; revised March 19, 2020 and April 30, 2020; accepted May 2, 2020. The work of Erdogan Aydin, Fatih Cogen, and Nihat Kabaoğlu was supported by Istanbul Medeniyet University under Project F-GAP-2018-1263. (Corresponding author: Erdogan Aydin.)

Fatih Cogen is with the Department of Mechatronics Systems Engineering, Turkish-German University, Istanbul 34820,Turkey (e-mail: cogen@ tau.edu.tr).

Erdogan Aydin and Nihat Kabaoglu are with the Department of Electrical and Electronics Engineering, Istanbul Medeniyet University, Istanbul 34857, Turkey (e-mail: erdogan.aydin@medeniyet.edu.tr; nihat.kabaoglu@medeniyet.edu.tr).

Ertugrul Basar is with the Department of Electrical and Electronics Engineering, Koç University, Istanbul 34450, Turkey (e-mail: ebasar@ku.edu.tr).

Haci Ilhan is with the Department of Electronics and Communications Engineering, Yıldız Technical University, Istanbul 34220, Turkey (e-mail: ilhanh@yildiz.edu.tr).

Digital Object Identifier 10.1109/JSYST.2020.2993704 rates [1]. Additionally, the tremendous number of users in 5G wireless networks is expected to increase energy consumption drastically. Thus, high priority has been given to the design of energy-efficient wireless communication systems with high data rates for device-to-device communications, spectrum sharing, ultradense networks, millimeter-wave networks, the Internet of Things communications, index modulation (IM), and multipleinput multiple-output (MIMO) systems [2]-[4].

MIMO systems, which provide significant improvements in channel capacity and error performance, form the basis of nextgeneration wireless communication systems. MIMO systems increase throughput and coverage area on one hand, whereas on the other hand, they provide capacity and diversity gains by exploiting multichannel capabilities [5], [6]. One of the important MIMO techniques that have emerged in recent years and also has become a focus of interest by researchers is the spatial modulation (SM) technique. In the SM scheme, while both the receiver and transmitter terminals are equipped with multiple antennas to obtain the spatial diversity of the rich scattering environment, only one antenna is activated at the transmitter terminal in each transmission time-slot. Consequently, the interantenna synchronization problem ceases to exist, the interchannel interference is prevented, and thus, the complexity of the communication system is greatly reduced. Also, in the SM scheme in addition to the transmitted data symbols, the active antenna indices of the transmitter are used to convey information. Thanks to this rational scheme, the data rate increases logarithmically along with the number of transmit antennas [7]. Thus, SM technique has recently been discussed in many academic studies [8]-[14], due to its high spectral efficiency, high performance, energy efficiency, and low complexity. In [8], researchers suggest a low complexity adaptive SM (ASM) technique based on the sphere search technique. The current ASM schemes examine all error vectors in the search area to find the most appropriate ASM mode. In [9], researchers are attempting to remove in-phase $(I)$ and quadrature $(Q)$ components' imbalance ( $I Q$ imbalance) causing spatial performance degradation of SM. For this reason, they recommend the most optimal maximum probability detector for SM-based transmission. In [11], a new approach to $\mathrm{SM}$ is presented. The main idea of the proposed approach is to change the active antenna in the middle of the symbol duration. The main advantages of this approach are the improved bit error rate (BER) performance and reduction in the number of transmitting antennas. In [12], researchers have proposed a new MIMO technique, called fully generalized SM (F-GSM). In this 
new system, the rate of data transmission along with the number of transmit antennas is increasing linearly, not logarithmically as in traditional SM techniques. Besides, F-GSM achieves a higher data rate than the conventional SM and the generalized-SM with a lower number of transmitting antennas. In [13], a technique called spatial permutation modulation (SPM) is proposed, which modulates the data bits into a permutation vector and activates the transmit antenna in successive time samples. SPM provides higher diversity gain and lowers BER compared to the conventional SM technique. Another technique is block-based SM (BSM) technique, changing the number of active antennas in each transmission vector, but keeping the total number of active antennas on a block constant. Based on this article, in [14], the spatial dimensions are better used, and the BSM scheme is generalized to obtain better BER.

Over the past few years, the IM scheme has been one of the most important research topics and has been shown as a promising candidate for next-generation wireless communication systems [15]. As can be understood by its name, IM techniques convey information in the indices of units, such as transmit antennas, subcarriers, time intervals, frequency carriers, space-time matrices, spreading codes, etc. In this technique, indices are transmitted by embedding them into the transmitted or received signals. Thus, little or no energy is spent on additional information carried in the indices. As a result, IM schemes provide excellent results in terms of both spectral and energy efficiency [15]-[17].

Direct sequence spread spectrum (DS-SS), one of the cornerstones of the generalized CIM-SM (GCIM-SM) technique uses orthogonal spreading codes (e.g., Walsh Hadamard Codes) or pseudo-noise sequences to transmit signals. It has many benefits such as secluded performance, jamming resistance, energy efficiency, sharing of the same channel with many users, noise and interference reduction, etc. Also, since CIM-aided IM techniques send the bits using orthogonal code indices with the spread spectrum technique, the extra bits carried in the code index will be less affected by the sensitivity of the communication channel. [18], [19]. The CIM spread spectrum (CIM-SS) system based on the DS-SS technique, which is included in studies [20]-[22], uses spreading code indices to convey extra information. In the CIM-SS scheme, a part of the information bits is used to transmit the modulated symbol, whereas the other part is used to select the spreading code indices. The selected spreading code is multiplied by the modulated symbols and transmitted without using any energy. In the receiver part, the spreading code index is determined with the aid of a simple correlator, and then, the received symbol is demodulated and the code is estimated. With this rational scheme, a part of the data bits are transmitted directly through the channel with the modulated signal, whereas the other part is carried by being embedded in the transmitted signal without spending any energy. For this reason, the CIM-SS system not only increases the data rate and efficiency of the system but also reduces the energy consumption [23], [24]. In [25], the CIM scheme is adapted to the quadrature SM (QSM) method.

In addition to these, works in [26] and [27] are rational and recently published IM techniques. In [26], a low power consumption and low operational complexity IM family, called joint code-frequency IM, has been proposed by the authors for multiuser communication systems. In [27], a new IM technique is proposed for optical communication systems based on the principle of driving the signal's time and frequency together. In [27], a frequency-shift keying modulated symbol carrying information (based on the multipulse position modulation technique) is sent during the active slots, rather than sending a single pulse shape that does not carry additional information.

\section{A. Contributions and Organization}

In this article, rational CIM and SM techniques are combined and generalized for $M$-QAM modulation to deal with the aforementioned energy-efficiency, error performance, and data-rate requirements. In the considered system, the information bits are mapped to spreading codes and antenna indices in addition to conventional constellation symbols.

As a result of the theoretical derivations and analyses, we can summarize the contribution of our article as follows.

1) CIM-SM technique presented in [23] and [24] has been generalized for the $M$-QAM. Contrary to the existing CIM-SM studies, in this article, we focus on the analyses to generalize the CIM-SM for the $M$-QAM. The CIM-SM technique is considered only for $\operatorname{BPSK}(M=2)$. Therefore, the number of bits carried in modulation has been limited to 2 b. Besides, in [23] and [24], the performance results are presented only by simulation.

2) Analytical expressions for average BER, energyefficiency, and throughput of the considered GCIM-SM system are derived.

3) BER performance results of the GCIM-SM system are compared with the CIM-QSM, DS-SS, CIM-SS, SM, and QSM techniques for the same number of bits per symbol and same symbol duration.

4) Simulation results prove that the GCIM-SM scheme provides better error performance than DS-SS, SM, QSM, and CIM-SS systems while consuming less transmission energy.

The remainder of the article is organized as follows. In Section II, we introduce the GCIM-SM scheme. In Section III, BER performance, energy-efficiency, throughput, and data rate analyses of the proposed system are presented. Simulation results and performance comparisons are given in Section IV. Finally, Section V concludes the article.

\section{SYSTEM MODEL}

The considered system model for the GCIM-SM technique is shown in Fig. 1. The system consists of $N_{T}$ transmit antennas, $N_{R}$ receive antennas and $2^{L}$ Walsh Hadamard codes. In the GCIM-SM system, the use of $M$-QAM modulation is envisaged because of its good error performance, but $M$-PSK modulation could be more suitable for implementations requiring lower energy consumption.

When the transmitter structure of the GCIM-SM system is taken into account, the vector $\mathbf{b}$ with dimensions $p \times 1$ is the binary information vector to be transmitted during the $T_{s}$ symbol period. Here, $p$ bits are subdivided into three blocks: $p_{1}=\log _{2}\left(N_{T}\right)$ bits, $p_{2}=\log _{2}(M)$ bits and $2 L$ bits such that $p=p_{1}+p_{2}+2 L$. For these blocks, $p_{2}$ bits select an $M$-QAM symbol, $2 L$ bits select spreading codes of the $I Q$-components, whereas $p_{2}+2 L$ bits are conveyed with both $I$ and $Q$ components, the $p_{1}$ bits are transmitted in the antenna indices of the SM. 


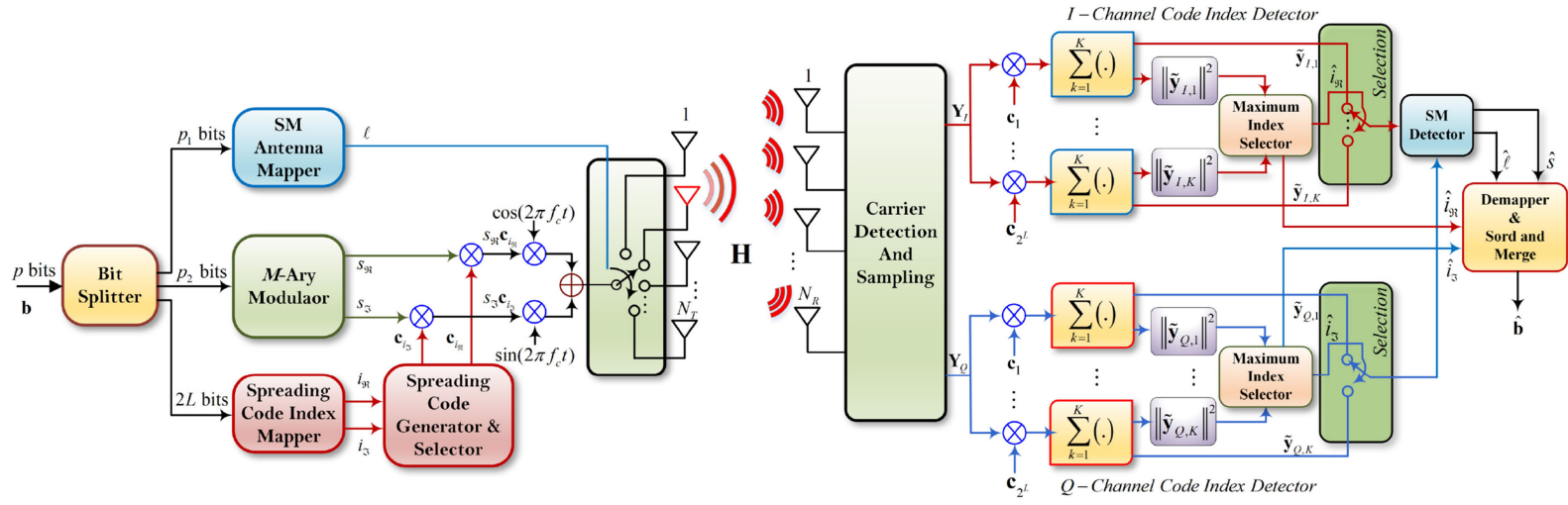

Fig. 1. System model of the GCIM-SM scheme.

Eventually, as can be seen from the transmitter of the GCIM-SM system, the spreaded symbol sequences are transmitted over the single antenna activated by the SM scheme.

The $M$-QAM modulated complex symbol can be given in general form as follows:

$$
s=s_{\Re}+j s_{\Im}
$$

where $s_{\Re}$ and $s_{\Im}$ are the real and imaginary components of $s$, respectively. $s_{\Re}$ and $s_{\Im}$ are selected according to the bit sequence of $p_{2}$. $s_{\Re}$ and $s_{\Im}$ are spreaded by the $\mathbf{c}_{i_{\Re}}$ and $\mathbf{c}_{i_{\Im}}$ spreading codes selected by the bits sequence of $2 L$, where spreading codes consist of \pm 1 . Consequently, the spreaded signals $s_{\Re} \mathbf{c}_{i_{\Re}}$ and $s_{\Im} \mathbf{c}_{i_{\Im}}$ are transmitted through the $I$ and $Q$ components from the $\ell$ th antenna activated by the bit sequence of $p_{1}$. From this point, the faded noisy signal received by the GCIM-SM receiver can be expressed as follows:

$$
\begin{aligned}
y(t) & =\sum_{k=1}^{K}\left(s_{\Re} c_{i_{\Re}, k} p\left(t-k T_{c}\right) \cos \left(2 \pi f_{c} t\right)\right. \\
& \left.+s_{\Im} c_{i_{\Im}, k} p\left(t-k T_{c}\right) \sin \left(2 \pi f_{c} t\right)\right) h_{\ell}(t)+n(t)
\end{aligned}
$$

where $p(t)$ is a unit rectangular pulse shaping filter on $\left[0, T_{c}\right]$ time period, $T_{c}$ is the chip duration of the spreading code, $h_{\ell}(t) \ell=$ $1,2, \ldots, N_{T}$, is the Rayleigh fading channel impulse response and $n(t) \sim C\left(0, N_{0}\right)$ is a complex Gaussian random process with zero mean and $N_{0} / 2$ variance per dimension.

Since, the $I$ and $Q$ components of the signal model have a similar structure, the signal model given in (2) can be rewritten separately for the $I$ and $Q$ components. Thus, after the perfect carrier detection and sampling at the output of the channel, the $k$ th noisy chip signal $(k=1,2, \ldots, K)$ of the $r$ th receive antenna $\left(r=1,2, \ldots, N_{R}\right)$ is given for both of the $I$ and $Q$ components would be as follows:

$$
\begin{aligned}
y_{I} & =s_{\Re} c_{i_{\Re}} h_{\ell}+n_{I} \\
y_{Q} & =s_{\Im} c_{i_{\Im}} h_{\ell}+n_{Q}
\end{aligned}
$$

where, to simplify the notation, we drop the argument $r$ and $k$. $\ell=1,2, \ldots, N_{T}$ and $i_{\Re}, i_{\Im}=1,2, \ldots, 2^{L} . h_{\ell}$ is the Rayleigh fading channel coefficient with zero mean and variance of $\sigma_{h}^{2}=1 . n_{I}$ and $n_{Q}$ are the AWGN noise terms of the $I$ and $Q$ components, respectively, and $n$ can be written as $n=n_{I}+n_{Q}$. Thus, the vectorial representation of (3) can be expressed as

$$
\begin{gathered}
\mathbf{Y}_{I}=s_{\Re} \mathbf{h}_{\ell} \mathbf{c}_{i_{\Re}^{T}}^{T}+\mathbf{N}_{I} \\
\mathbf{Y}_{Q}=s_{\Im} \mathbf{h}_{\ell} \mathbf{c}_{i_{\Im}}^{T}+\mathbf{N}_{Q}
\end{gathered}
$$

where $\mathbf{Y}_{I}=\left[\mathbf{y}_{I, 1} \ldots \mathbf{y}_{I, K}\right]$ and $\mathbf{Y}_{Q}=\left[\mathbf{y}_{Q, 1} \ldots \mathbf{y}_{Q, K}\right]$, here $\mathbf{y}_{I, k}$ and $\mathbf{y}_{Q, k}$ are the $k$ th column of $\mathbf{Y}_{I}$ and column of $\mathbf{Y}_{Q}$, respectively. $\mathbf{h}_{\ell}$ is the $\ell$ th column of $\mathbf{H}$, which is the channel matrix with dimensions $N_{R} \times N_{T}$. The rows of $\mathbf{H}$ carry the receiver antenna indices information, whereas the columns carry the transmitter antenna indices information. $\mathbf{H}$ can be expressed as $\mathbf{H}=\left[\mathbf{h}_{1} \mathbf{h}_{2} \ldots \mathbf{h}_{N_{T}}\right]$ and also $\mathbf{c}_{i_{\Re}}$ and $\mathbf{c}_{i_{\Im}}$ with the size of $K \times 1$ are the Walsh Hadamard spreading codes. $\mathbf{N}_{I}$ and $\mathbf{N}_{Q}$ are the AWGN matrix.

As shown in Fig. 1, after the despreading operation at the receiver terminal for each of the $I$ and $Q$ components, each of the branches is used to estimate the spreading code indices, the active antenna indices, and the transmitted symbol sequences, i.e., $\left(\hat{i}_{\Re}, \hat{i}_{\Im}, \hat{\ell}, \hat{s}\right)$. Here, to reduce the complexity of the GCIMSM system, detection of $\hat{\ell}$ and $\hat{s}$ will be performed by the SM detector after the estimates of Walsh Hadamard code indices $\left(\hat{i}_{\Re}, \hat{i}_{\Im}\right)$ are obtained. Therefore, using the selection block as shown at the receiver side, the estimated $\left(\hat{i}_{\Re}, \hat{i}_{\Im}\right)$ indices are fed back to the despreaded vector set. Then, only the $\mathbf{y}_{\hat{i}_{\Re}}$ and $\mathbf{y}_{\hat{i}_{\Im}}$ despreaded data vectors that are associated with the $\left(\hat{i}_{\Re}, \hat{i}_{\Im}\right)$ indices are applied to the input of the SM detector. In this way, the complexity of the system is greatly reduced. For this reason, first, the spreading code indices are estimated from the sampled signal using correlator branches. To do this, the matrices $\mathbf{Y}_{I}$ and $\mathbf{Y}_{Q}$ are multiplied by the corresponding spreading code $\mathbf{c}_{i}$ in each branch and summed over the period $T_{s}=K T_{c}$. Thus, the despreaded output of the $i$ th correlator for both the $I$ and $Q$ components can be expressed as follows:

$$
\begin{aligned}
\tilde{\mathbf{y}}_{I, i} & =\mathbf{Y}_{I} \mathbf{c}_{i}=\left[\begin{array}{lll}
\mathbf{y}_{I, 1} & \mathbf{y}_{I, 2} \ldots \mathbf{y}_{I, K}
\end{array}\right] \mathbf{c}_{i} \\
\tilde{\mathbf{y}}_{Q, i} & =\mathbf{Y}_{Q} \mathbf{c}_{i}=\left[\begin{array}{l}
\mathbf{y}_{Q, 1} \mathbf{y}_{Q, 1} \ldots \mathbf{y}_{I, K}
\end{array}\right] \mathbf{c}_{i}
\end{aligned}
$$

where $i=1,2, \ldots, 2^{L}$. From this point forth, the $r$ th element of $\tilde{\mathbf{y}}_{I, i}$ and $\tilde{\mathbf{y}}_{Q, i}$ can be, respectively, expressed as follows: 


$$
\begin{aligned}
\tilde{y}_{I, i} & =\sum_{k=1}^{K} c_{i, k} y_{I, k}=\sum_{k=1}^{K} c_{i, k}\left(s_{\Re} c_{i_{\Re}, k} h_{\ell}+n_{I, k}\right) \\
& = \begin{cases}s_{\Re} h_{\ell} E_{c}+\tilde{n}_{I}, & \text { if } i=\hat{i}_{\Re} \\
\tilde{n}_{I}, & \text { if } i \neq \hat{i}_{\Re}\end{cases} \\
\tilde{y}_{Q, i} & =\sum_{k=1}^{K} c_{i, k} y_{Q, k}=\sum_{k=1}^{K} c_{i, k}\left(s_{\Im} c_{i_{\Im}, k} h_{\ell}+n_{Q, k}\right) \\
& = \begin{cases}s_{\Im} h_{\ell} E_{c}+\tilde{n}_{Q}, & \text { if } i=\hat{i}_{\Im} \\
\tilde{n}_{Q}, & \text { if } i \neq \hat{i}_{\Im}\end{cases}
\end{aligned}
$$

where $E_{c}$ is the average energy transmitted per spreading code and is expressed as $E_{c}=\frac{1}{\sqrt{K}} \sum_{k=1}^{K} c_{i, k}^{2}$. Also, $\tilde{n}_{I}=$ $\sum_{k=1}^{K} c_{i, k} n_{I, k}$ and $\tilde{n}_{Q}=\sum_{k=1}^{K} c_{i, k} n_{Q, k}$ are the correlated AWGN terms multiplied by the Walsh Hadamard code. It can be shown from (6) and (7) that the mean of $\tilde{y}_{I, i}$ is $s_{\Re} h_{\ell} E_{c}$ while $i=\hat{i}_{\Re}$ or 0 while $i \neq \hat{i}_{\Re}$, and the mean of $\tilde{y}_{Q, i}$ is $s_{\Im} h_{\ell} E_{c}$ while $i=\hat{i}_{\Im}$ or 0 while $i \neq \hat{i}_{\Im}$. Additionally, the variances can be written as: $\sigma_{\tilde{y}_{I, i}}^{2}=\sigma_{\tilde{y}_{Q, i}}^{2}=E_{c} N_{0}$. When despreading operation is performed through all despreader for both of the $I$ and $Q$ components, the resulting vector set at the receiver can be expressed with the help of (5) as follows:

$$
\begin{aligned}
\mathscr{Y}_{I} & =\left\{\tilde{\mathbf{y}}_{I, 1}, \tilde{\mathbf{y}}_{I, 2}, \ldots, \tilde{\mathbf{y}}_{I, 2^{L}}\right\} \\
\gamma_{Q} & =\left\{\tilde{\mathbf{y}}_{Q, 1}, \tilde{\mathbf{y}}_{Q, 2}, \ldots, \tilde{\mathbf{y}}_{Q, 2^{L}}\right\} .
\end{aligned}
$$

To estimate the indices of the spreading codes for $I$ and $Q$ components in the system, first, the squared norm of the vectors $\left\{\left\|\tilde{\mathbf{y}}_{I, i}\right\|^{2}\right\}_{i=1}^{2 L}$ in the set of $Y_{I}$ and $\left\{\left\|\tilde{\mathbf{y}}_{Q, i}\right\|^{2}\right\}_{i=1}^{2^{L}}$ in the set of $\Upsilon_{Q}$ are taken, and then, the indices of the maximum elements in the sets are determined. Since the Walsh Hadamard codes are orthogonal to each other, i.e., $\sum_{k=1}^{K} c_{i, k} c_{j, k}=\left\{\begin{array}{l}1, \text { if } i=j \\ 0, \text { if } i \neq j\end{array}\right.$, the largest-valued element of the normed vector is also equal to the element over the same indices. From this point forth, the indices of the maximum elements of the normed vector sets can be determined as follows:

$$
\begin{aligned}
& \hat{i}_{\Re}=\underset{i}{\arg \max }\left\{\left\|\tilde{\mathbf{y}}_{I, i}\right\|^{2}\right\} \\
& \hat{i}_{\Im}=\underset{i}{\arg \max }\left\{\left\|\tilde{\mathbf{y}}_{Q, i}\right\|^{2}\right\} .
\end{aligned}
$$

Once $\hat{i}_{\Re}$ and $\hat{i}_{\Im}$ are obtained by the spreading code index detectors, the maximum likelihood (ML)-based SM detector will consider all of the combinations of the $(\ell, s)$ over the despreaded signals $\tilde{\mathbf{y}}_{I, \hat{i}_{\Re}}$ and $\tilde{\mathbf{y}}_{Q, \hat{i}_{\Im}}$ associated with the $\left(\hat{i}_{\Re}, \hat{i}_{\Im}\right)$ indices to obtain the estimations of $\hat{\ell}$ and $\hat{s}_{d \mathrm{f}}$. Thus, the ML estimates of the $\left(\ell, s_{d x}\right)$ parameters for both of the $I$ and $Q$ components of the GCIM-SM system can be expressed as follows:

$$
\begin{aligned}
{[\hat{\ell}, \hat{s}] } & =\underset{\ell, s}{\operatorname{argmin}}\left\{\left\|\left(\tilde{\mathbf{y}}_{I, \hat{i}_{\Re}}+j \tilde{\mathbf{y}}_{Q, \hat{i}_{\Im}}\right)-E_{c} s \mathbf{h}_{\ell}\right\|^{2}\right\} \\
& =\underset{\ell, s}{\operatorname{argmin}}\left\{\left\|\xi_{\ell}\right\|^{2}-2 \operatorname{Re}\left\{\left(\tilde{\mathbf{y}}_{I, \hat{i}_{\Re}}+j \tilde{\mathbf{y}}_{Q, \hat{i}_{\Im}}\right)^{H} \xi_{\ell}\right\}\right\}
\end{aligned}
$$

where $\xi_{\ell}=E_{c} s_{d} \mathbf{h}_{\ell}$.

Finally, with the bit-back matching technique using the detected $\left(\hat{i}_{\Re}, \hat{i}_{\Im}, \hat{\ell}, \hat{s}\right)$ values, the originally transmitted bit sequence $\hat{b}$ is reconstructed at the receiver with the help of demapper as shown at the receiver of the GCIM-SM system.

\section{Performance Analysis of the GCIM-SM System}

In this section, BER, throughput, data rate, and energy efficiency analyses of the GCIM-SM system are evaluated and, then, compared with the SM, QSM, CIM-SS, and conventional DS-SS techniques.

\section{A. BER Analysis of the GCIM-SM System}

As shown in the structure of GCIM-SM, and also previously mentioned, the incoming binary information data bits are divided into three parts. Therefore, the total BER expression of the considered system, which is defined as $\mathbb{P}_{\mathrm{GCIM}-\mathrm{SM}}$, can be expressed as a function of the bit error probability (BEP) of two error events. That is to say, the total BER occurring at the receiver is the sum of the BEP of the spreading code indices' mapped bits $\left(\mathbb{P}_{1}\right)$ and the BEP of the active antenna indices and modulated bits $\left(\mathbb{P}_{2}\right)$. Consequently, the average BER for the GCIM-SM system can be defined as follows:

$$
\mathbb{P}_{\mathrm{GCIM}-\mathrm{SM}}=\frac{2 L}{p} \mathbb{P}_{1}+\frac{p_{1}+p_{2}}{p} \mathbb{P}_{2}
$$

The error events $\mathbb{P}_{1}$ and $\mathbb{P}_{2}$ depend on the $I$ and $Q$ components' average probability of erroneous spreading code indices detection $\mathbb{P}_{c}^{\hat{i}_{R}}$ and $\mathbb{P}_{c}^{\hat{i}_{3}}$. Therefore, the correct reception of SM modulated symbols relies on the correct detection of the spreading code indices at the receiver side of Fig 1. In the GCIM-SM system, two different types of errors may occur. In the first case, the $\hat{i}_{\Re}$ and $\hat{i}_{\Im}$ are correctly detected (i.e., there is no error in the code indices' mapped bits), but the SM detector makes incorrect decisions. In the second case, $\hat{i}_{\Re}$ and $\hat{i}_{\Im}$ are detected incorrectly, and therefore, the SM detector attempts to detect bits based on the output of the incorrectly selected despreader (i.e., $\tilde{\mathbf{y}}_{I, i \neq \hat{i}_{\tilde{r}}}$, $\left.\tilde{\mathbf{y}}_{Q, i \neq \hat{i}_{3}}\right)$. In this case, since the receiver has no chance of making a correct decision, the probability of bit error is straightforwardly equal to 0.5. Consequently, the BER expression for antenna indices and modulated bits can be expressed as follows:

$$
\mathbb{P}_{2}=\mathbb{P}_{\text {SM }}\left(1-\mathbb{P}_{\mathrm{c}}\right)+\frac{1}{2} \mathbb{P}_{\mathrm{c}}
$$

where $\mathbb{P}_{S M}$ is the BEP for the SM detector and $\mathbb{P}_{c}=\frac{1}{2} \mathbb{P}_{c}^{\hat{i}_{\aleph_{R}}}+$ $\frac{1}{2} \mathbb{P}_{c}^{\hat{i}_{\Im}}$.

The BEP expression of the spreading code indices' mapped bits $\mathbb{P}_{1}$ can be defined by the following two values: $\mathbb{P}_{c}$ and $L$. If the pair of code indices $\left(\hat{i}_{\Re}, \hat{i}_{\Im}\right)$ is erroneously estimated, the combination of original spreading code mapping bits will be erroneously detected. Therefore, each erroneous combination will result in the detection of a different number of erroneous bits compared to original spreading code mapping bits. Consequently, after some intermediate steps, the BER for spreading code indices' bits $\mathbb{P}_{1}$ can be expressed as follows:

$$
\mathbb{P}_{1}=\frac{\mathbb{P}_{\mathrm{c}}}{L} .
$$




\section{B. Average Probability of Erroneous Code-Index Detection}

As depicted in the $I$ and $Q$ channels' code index detectors of the receiver of the GCIM-SM system and also can be seen from (10) and (11); since $\mathbb{P}_{\mathrm{c}}$ is a function of $\mathbb{P}_{\mathrm{c}}^{\hat{i}_{\mathfrak{R}}}$ and $\mathbb{P}_{\mathrm{c}}^{\hat{i}_{\mathfrak{S}}}, \mathbb{P}_{\mathrm{c}}^{\hat{i}_{\mathfrak{R}}}$ and $\mathbb{P}_{c}^{\hat{i}_{\Im}}$ should be determined first.

The probability of the $\mathbb{P}_{c}^{\hat{i}_{\Re}}$ or $\mathbb{P}_{c}^{\hat{i}_{\Im}}$ can be defined as the probability that the element with the largest norm value (i.e., $\left\|\tilde{\mathbf{y}}_{I, \hat{i}_{\Re}}\right\|^{2}$ or $\left.\left\|\tilde{\mathbf{y}}_{Q, \hat{i}_{S}}\right\|^{2}\right)$ is less than the minimum norm value

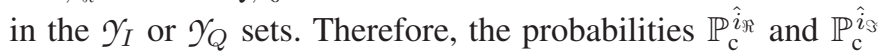
conditioned on the spreading code and channel coefficients for equiprobable transmitted spreading codes can be defined as

$$
\begin{aligned}
& \mathbb{P}_{\mathrm{c}}^{\hat{i}_{\Re}}=\operatorname{Pr}\left(\left\|\tilde{\mathbf{y}}_{I, \hat{i}_{\Re}}\right\|^{2} \underset{i \in\left\{1,2, \ldots, 2^{L}\right\}, i \neq \hat{i}_{\Re}}{<}\left\{\left\|\tilde{\mathbf{y}}_{I, i}\right\|^{2}\right\} \mid \mathbf{c}_{i}, \mathbf{h}_{\ell_{\Re}}\right) \\
& \mathbb{P}_{\mathrm{c}}^{\hat{i}_{\Im}}=\operatorname{Pr}\left(\left\|\tilde{\mathbf{y}}_{Q, \hat{i}_{\Im}}\right\|^{2} \underset{i \in\left\{1,2, \ldots, 2^{L}\right\}, i \neq \hat{i}_{\Im}}{<}\left\{\left\|\tilde{\mathbf{y}}_{Q, i}\right\|^{2}\right\} \mid \mathbf{c}_{i}, \mathbf{h}_{\ell_{\Im}}\right)
\end{aligned}
$$

where $\quad \tilde{\mathbf{y}}_{I, \hat{i}_{\Re}}=E_{c} \mathcal{S}_{\Re} \mathbf{h}_{\ell_{\Re}}+\tilde{\mathbf{n}}_{I, \hat{i}_{\Re}}, \quad \tilde{\mathbf{y}}_{Q, \hat{i}_{\Im}}=E_{c} \mathcal{s}_{\Im} \mathbf{h}_{\ell_{\Im}}+\tilde{\mathbf{n}}_{Q, \hat{i}_{\Im}}$, and $\tilde{\mathbf{y}}_{I, i}=\tilde{\mathbf{n}}_{I, i}$ with $i \neq \hat{i}_{\Re}, \tilde{\mathbf{y}}_{Q, i}=\tilde{\mathbf{n}}_{Q, i}$ with $i \neq \hat{i}_{\Im}$. It can be easily shown from (16) and (17) that the $\mathbb{P}_{\mathrm{c}}=\mathbb{P}_{\mathrm{c}}^{\hat{i}_{\Re}}=\mathbb{P}_{\mathrm{c}}^{\hat{i}_{\Im}}$ for the $I$ and $Q$ channels' spreading code indices detection. Therefore, calculating the probability of any channel is sufficient to know the other. It would, therefore, be reasonable to continue with the possibility of detecting erroneous $I$-channel spreading code indices detection. Since each noise sample at the receiver is multiplied with a different spreading code, the norm squared random variables (RVs) are independent and follow noncentral chi-square distribution (non-CCSD) while $i=\hat{i}_{\Re}$ and CCSD while $i \neq \hat{i}_{\Re}$ with even $N_{R}$-degrees of freedom (DoF) [28].

Finally, to obtain $\mathbb{P}_{\mathrm{c}}$ for the GCIM-SM scheme, the order statistics theory is used to derive $I$ and $Q$ spreading code index probabilities. For this purpose, let $\Upsilon$ and $\Psi$ be two RVs and defined as follows:

$$
\begin{aligned}
& \Upsilon=\min \left\{\Upsilon_{i}\right\}, \quad i \in\left\{1,2, \ldots, 2^{L}\right\}, i \neq \hat{i}_{\Re} \\
& \Psi=\left\|\tilde{\mathbf{y}}_{I, \hat{i}_{\Re}}\right\|^{2}
\end{aligned}
$$

where $\Upsilon_{i}=\left\|\tilde{\mathbf{y}}_{i}^{I}\right\|^{2}$. When (18) is taken into account, the RV of $\Upsilon_{i}$ follows CCSD while $\Psi$ has the non-CCSD. The probability density function (pdf) of $\Psi$ and cumulative distribution function of $\Upsilon_{i}$ with even $N_{R}$-DoF non-CCSD are, respectively, expressed by $[28,(2.3-29,2.3-24)]$

$$
\begin{aligned}
& f_{\Psi}(\psi)=\frac{1}{2 \sigma_{\Psi}^{2}}\left(\frac{\psi}{\mu_{\hat{i}_{\Re}}^{2}}\right)^{\frac{N_{R}-2}{4}} e^{-\frac{\mu_{\hat{i}_{R}}^{2}+\psi}{2 \sigma_{\Psi}^{2}}} I_{\frac{N_{R}}{2}-1}\left(\frac{\mu_{\hat{i}_{\Re}}}{\sigma_{\Psi}^{2}} \sqrt{\psi}\right), \Psi>0 \\
& F_{N_{R}=2 z}\left(v_{i}\right)=1-e^{-\frac{v_{i}}{2 \sigma_{\Upsilon_{i}}^{2}}} \sum_{m=0}^{z-1} \frac{1}{m !}\left(\frac{v_{i}}{2 \sigma_{\Upsilon_{i}}^{2}}\right)^{m}, \Upsilon_{i}>0
\end{aligned}
$$

where $I_{\beta}(y)=\sum_{v=0}^{\infty} \frac{(y / 2)^{\beta+2 v}}{v ! \Gamma(\beta+v+1)}, y \geq 0$ is the modified Bessel function of the first kind and order $\beta$ [28, (2.3-31)], where $\Gamma(x)$ is the gamma function expressed as $\Gamma(x)=\int_{0}^{\infty} t^{x-1} e^{-x} d t$
[28, (2.3-22)]. $\mu_{\hat{i}_{\Re}}=\sqrt{\sum_{r=1}^{N_{R}}\left|E_{c} s_{d_{\Re}} h_{r, \ell_{\Re}}\right|^{2}} \quad$ [28, (2.3-30)], $\sigma_{\Upsilon_{i}}^{2}=2 N_{R}\left(E_{c} N_{0}\right)^{2}[28,(2.3-25)]$ and $\sigma_{\Psi}^{2}=2 N_{R}\left(E_{c} N_{0}\right)^{2}+$ $4 E_{c} N_{0} \mu^{2}[28,(2.3-40)]$. As a result, the probability of $\Psi<\Upsilon$ can be written with the aid of order statistics as

$$
\begin{aligned}
\operatorname{Pr}(\Psi<\Upsilon) & =\int_{0}^{\infty} \operatorname{Pr}(\psi<\Upsilon) f_{\Psi}(\psi) d \psi \\
& =\int_{0}^{\infty} \prod_{i=1}^{2^{L}-1} \operatorname{Pr}\left(\psi<\Upsilon_{i}\right) f_{\Psi}(\psi) d \psi
\end{aligned}
$$

By using (21), the probability of erroneous code indices' detection $\mathbb{P}_{\mathrm{c}}^{\hat{i}_{\mathcal{R}}}$ can be expressed as

$$
\begin{aligned}
& \mathbb{P}_{\mathrm{c}}^{\hat{i}_{\Re}}=\frac{1}{2 \sigma_{\Psi}^{2}} \int_{0}^{\infty}\left[e^{-\frac{v_{i}}{2 \sigma_{\Upsilon_{i}}^{2}}} \sum_{m=0}^{z-1} \frac{1}{m !}\left(\frac{v_{i}}{2 \sigma_{\Upsilon_{i}}^{2}}\right)^{v}\right]^{\left(2^{L}-1\right)} \\
& \times\left(\frac{\psi}{\mu_{\hat{i}_{\Re}}^{2}}\right)^{\frac{N_{R}-2}{4}} e^{-\frac{\mu_{\hat{i}_{\Re}}^{2}+\psi}{2 \sigma_{\Psi}^{2}}} I_{\frac{N_{R}}{2}-1}\left(\frac{\mu_{\hat{i}_{\Re}}}{\sigma_{\Psi}^{2}} \sqrt{\psi}\right) d \psi .
\end{aligned}
$$

Moreover, when (22) is considered carefully, it is seen that $\mathbb{P}_{\mathrm{c}}^{\hat{i}_{\Re}}$ is still dependent on the RV of $\mu_{\hat{i}_{\Re}}$ due to the RV of the Rayleigh fading channel gain. Thus, to obtain $\mathbb{P}_{c}, \mathbb{P}_{c}^{\hat{P}_{\aleph_{R}}}$ should be integrated over pdf of the $\mu_{\hat{i}_{\Re}}$. For this reason, let $\chi_{r} \triangleq$ $\left|s_{d_{\Re}} h_{r, \ell_{R}} E_{c}\right|$ be an RV. The mean and variance of $\chi_{r}$ are zero and $\sigma_{\chi_{r}}^{2}=\left(s_{d_{\Re}} E_{c}\right)^{2} \sigma_{h}^{2}$, respectively. Since $\mu_{\hat{i}_{\Re}}=\sqrt{\sum_{r=1}^{N_{R}} \chi_{r}^{2}}$, the pdf of $\mu_{\hat{i}_{\Re}}$ has a generalized Rayleigh distribution with the pdf of $f\left(\mu_{\hat{i}_{\Re}}\right)=\frac{\mu_{\hat{i}_{R}}^{N_{R^{-1}}}}{2^{\frac{N_{R}-2}{2}} \sigma_{\chi r}^{N_{R}} \Gamma\left(\frac{N_{R}}{2}\right)} e^{-\mu_{\hat{i}_{\Re}}^{2} / 2 \sigma_{\chi r}^{2}}, \mu_{\hat{i}_{\Re}} \geq 0$ [28, (2.3-52)]. Eventually, the average probability of erroneous code-index detection of the GCIM-SM scheme can be given as follows:

$$
\mathbb{P}_{\mathrm{c}}=\int_{0}^{\infty} \mathbb{P}_{\mathrm{c}}^{\hat{\mathrm{P}}_{\Re}} f \mu\left(\mu_{\hat{i}_{\Re}}\right) d \mu_{\hat{i}_{\Re}} .
$$

The integration of $\mathbb{P}_{c}$ expression can be easily obtained with the software that can do numerical integration, such as Mathematica and MATLAB.

\section{Probability of Bit Error of Active Antenna Indices and Modulated Bits}

The BEP analysis of the active antenna indices' and the modulated bits' error event for the GCIM-SM scheme $\mathbb{P}_{2}$, which is previously given in (14), will be performed in this section. The probability of erroneous code indices' detection $\left(\mathbb{P}_{\mathrm{c}}\right)$ in (14) was derived in (23). Therefore, in order to calculate $\mathbb{P}_{2}$, the average BEP for $\mathbb{P}_{\text {SM }}$ must be calculated. Accordingly, using the well-known union bounding technique as in [28], the expression of $\mathbb{P}_{\mathrm{SM}}$ can be given as follows [29]:

$$
\mathbb{P}_{\mathrm{SM}} \leq \frac{1}{2^{\tilde{p}}} \sum_{i=1}^{2^{\tilde{p}}} \sum_{j=1}^{2^{\tilde{p}}} \frac{\mathbb{P}_{\mathrm{e}}\left(\xi_{i} \rightarrow \hat{\xi}_{j}\right) N(i, j)}{\tilde{p}}
$$

where $\tilde{p}=p_{1}+p_{2}$ is the number of bits transmitted in active antenna indices and modulated symbols, $N(i, j)$ is expressed as the number of bit errors associated with the corresponding pairwise error event, and $\mathbb{P}_{\mathrm{e}}\left(\xi_{i} \rightarrow \hat{\xi}_{j}\right)$ is the average pairwise 
TABLE I

ENERgY-SAVING Comparisons of GCIM-SM SCHEME In PERCENTAGE COMPARED TO DS-SS, SM, QSM, AND CIM-SS SYSTEMS ( $\left.\mathcal{E}_{\mathrm{SAV}} \%\right)$

\begin{tabular}{||c|c|c||c|c|c|c||}
\hline$N_{T}$ & $M$ & $L$ & DS-SS & SM & QSM & CIM-SS \\
\hline \hline 2 & 4 & 4 & $83.33 \%$ & $72.72 \%$ & $63.63 \%$ & $9.09 \%$ \\
\hline 4 & 4 & 4 & $87.5 \%$ & $66.66 \%$ & $50 \%$ & $16.66 \%$ \\
\hline 8 & 8 & 5 & $81.25 \%$ & $62.5 \%$ & $43.75 \%$ & $18.75 \%$ \\
\hline
\end{tabular}

error probability, which can be expressed as

$$
\mathbb{P}_{\mathrm{e}}\left(\xi_{i} \rightarrow \hat{\xi}_{j}\right)=\mathcal{U}_{\alpha}^{N_{R}} \sum_{r=0}^{N_{R}-1}\left(\begin{array}{c}
N_{R}-1+r \\
r
\end{array}\right)\left[1-\mathcal{U}_{\alpha}\right]^{r}
$$

where $\mathcal{U}_{\alpha}=\frac{1}{2}\left(1-\sqrt{\frac{\sigma_{\alpha}^{2}}{1+\sigma_{\alpha}^{2}}}\right)$. Here, $\sigma_{\alpha}^{2}$ is given by [29]

$$
\sigma_{\alpha}^{2}=\left\{\begin{array}{l}
\frac{E_{c}}{2 N_{0}} \sigma_{h}^{2}\left(\left|s_{d}\right|^{2}+\left|\hat{s}_{d}\right|^{2}\right) \text { if } \mathbf{h}_{\ell} \neq \hat{\mathbf{h}}_{\ell} \\
\frac{E_{c}}{2 N_{0}} \sigma_{h}^{2}\left(\left|s_{d}-\hat{s}_{d}\right|^{2}\right) \text { if } \mathbf{h}_{\ell}=\hat{\mathbf{h}}_{\ell} .
\end{array}\right.
$$

Consequently, by substituting (24), (23), (15), and (14) into (13), we obtain the analytical average total probability of the error for the GCIM-SM system.

\section{ENERGY EFFICIENCY, THROUGHPUT, AND DATA RATE ANALYSIS}

In this section, we have conducted energy efficiency, throughput, and data rate analyses for the GCIM-SM scheme. First, the percentage of energy saving obtained through the extra bits, which are conveyed as a result of using the spreading codes and the active antenna indices is examined. Thereafter, we discuss the throughput and data rate analysis for the GCIM-SM system.

\section{A. Energy Efficiency Analysis}

In the GCIM-SM transmission scheme, $p_{2}$ bits are conveyed over directly by the modulated symbol, $2 L$ and $p_{1}$ bits are transmitted through the spreading code indices and the active antenna indices, respectively. Hence, $p_{1}+2 L$ bits transmission energy is not directly consumed by the system but it is carried with the spreading code and active antenna indices. As a result, according to the GCIM-SM scheme, the percentage of energy-saving $\left(\mathcal{E}_{\text {sav }}\right)$ per $p$ bits can be written as follows:

$$
\mathcal{E}_{\text {sav }}=\left(1-\frac{p_{2}}{p}\right) E_{b} \%
$$

where $E_{b}$ is the bit energy, which is defined as $\frac{E_{c}}{p}$. In Table I, the percentage of energy-saving comparisons compared to DS-SS, SM, QSM, and CIM-SS techniques for various $N_{T}, M$, and $L$ values are presented. For instance, when the number of bits transmitted at the same time period is selected as $p=12$, $\mathcal{E}_{\text {sav }}$ of the GCIM-SM system compared to the DS-SS, SM, QSM, and CIM-SS systems is $87.5 \%, 66.66 \%, 50 \%$, and $16.16 \%$, respectively. Considering Table I, it is seen that the GCIM-SM system is reasonably energy efficiency scheme compared to DS-SS, SM, QSM, and CIM-SS systems.
TABLE II

DATA RATE COMPARISONS (BITS PER $T_{S}$ )

\begin{tabular}{||c|c|c||c|c|c|c|c||}
\hline$N_{T}$ & $M$ & $L$ & GCIM-SM & DS-SS & SM & QSM & CIM-SS \\
\hline \hline 2 & 4 & 3 & 9 & 2 & 3 & 4 & 8 \\
\hline 4 & 4 & 4 & 12 & 2 & 4 & 6 & 10 \\
\hline 8 & 8 & 5 & 16 & 3 & 6 & 9 & 13 \\
\hline
\end{tabular}

\section{B. System Complexity Analysis}

The complexity of the GCIM-SM scheme can be calculated by the number of operations required to perform the transmission. At the transmitter side and the receiver side, two spreading operations $(2 K)$ are performed to transmit one modulated symbol for $I Q$-channel and $\left(N_{R}+1\right)$ complex operations for each $Y^{I}$ and $\gamma^{Q}$, and $8 N_{R} 2^{p}$ reel multiplications (RMs) for the SM to search ML over $p$-dimensional space diagram of (12) [29]. Consequently, the overall complexity can be expressed as $\mathcal{O}_{\text {GCIM-SM }}=$ $2 K+16 K 2^{L}\left(N_{R}+1\right)+8 N_{R} 2^{p}$. Similarly, the complexity of CIM-QSM and SM are $\mathcal{O}_{\text {CIM-QSM }}=2 K+16 K 2^{L}\left(N_{R}+1\right)+$ $8 N_{R} 2^{p+p_{1}}$ and $8 N_{R} 2^{m_{\mathrm{SM}}} \mathrm{RMs}$, where $m_{\mathrm{SM}}=p_{1}+p_{2}$ [29]. However, to transmit $p$ bits, the total system complexity of the $\mathrm{SM}$ technique is $\mathcal{O}_{\mathrm{SM}}\left(1+\frac{2 L}{p_{1}+p_{2}}\right)$.

\section{Throughput and Data Rate}

The throughput of a communication system is usually expressed as the number of correct bits that a unit has obtained per unit time. Thus, the throughput of GCIM-SM scheme can be given as follows [30]:

$$
R_{t}=\frac{\left(1-\mathbb{P}_{\mathrm{GCIM}-\mathrm{SM}}\right)}{T_{s}} p
$$

where $\left(1-\mathbb{P}_{\mathrm{GCIM}-\mathrm{SM}}\right)$ is the probability of the correct bits received throughout the symbol duration $T_{s}$. Since DS-SS, SM, QSM, and CIM-SS techniques have the same $T_{s}$ symbol duration, the throughput comparisons can be done fairly. In Table II, data rate comparisons for various $N_{T}, M$, and $L$ values are presented. For instance, during the same symbol duration $T_{s}$ and while $N_{T}=4, M=4$, and $L=4$, the GCIM-SM system transmits $12 \mathrm{~b}$, whereas the DS-SS, SM, QSM, and CIM-SM schemes transmit 2, 4, 6, and $10 \mathrm{~b}$, respectively.

\section{SimUlation RESUltS AND DisCUSSION}

In this section, computer simulation results are presented and compared in the presence of Rayleigh fading channels for GCIM-SM, CIM-SS, DS-SS (with $M$-PSK/QAM), QSM, and SM techniques. In the receiver of the GCIM-SM system, initially, the spreading code indices of the $I$ - and $Q$-component from the received signal are obtained by the correlators. Afterward, the ML detector is used to estimate the transmitted symbols and indices. The SNR used in the simulations herein is defined as $\operatorname{SNR}(\mathrm{dB})=10 \log _{10}\left(E_{s} / N_{0}\right)$, where $E_{s}=$ $\sum_{k=1}^{K}\left(c_{i, k} / \sqrt{K}\right)^{2}$ is the average symbol energy. For the performance comparisons, $K$ is chosen as 32 for Figs. 2-4, whereas $K=64$ for Figs. 5 and 6.

In Figs. 2 and 3, BER performance curves of GCIM-SM, CIM-SS, QSM, SM, and DS-SS techniques for $p=9 \mathrm{~b}$ are presented when $N_{R}=1$ and $N_{R}=4$, respectively. For Figs. 2 


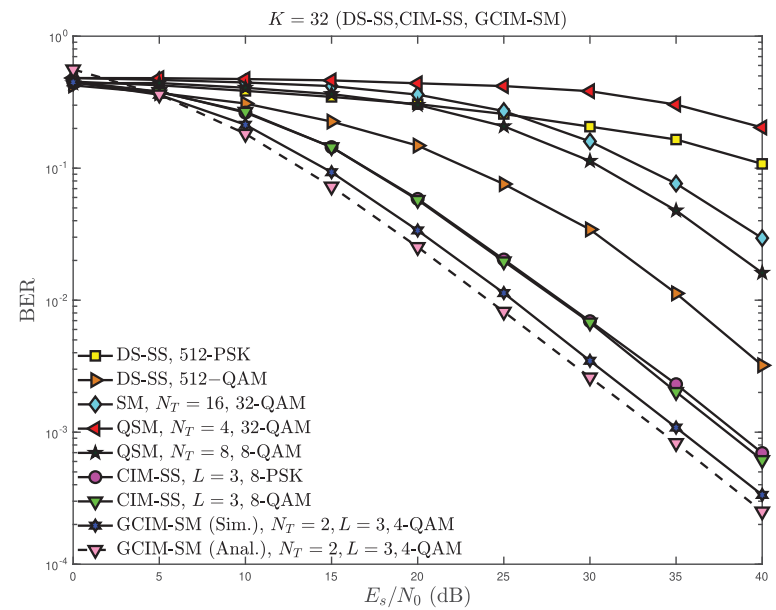

Fig. 2. Performance comparisons of GCIM-SM, CIM-SS, QSM, SM, and DS-SS systems for $p=9 \mathrm{~b}$ when $N_{R}=1$.

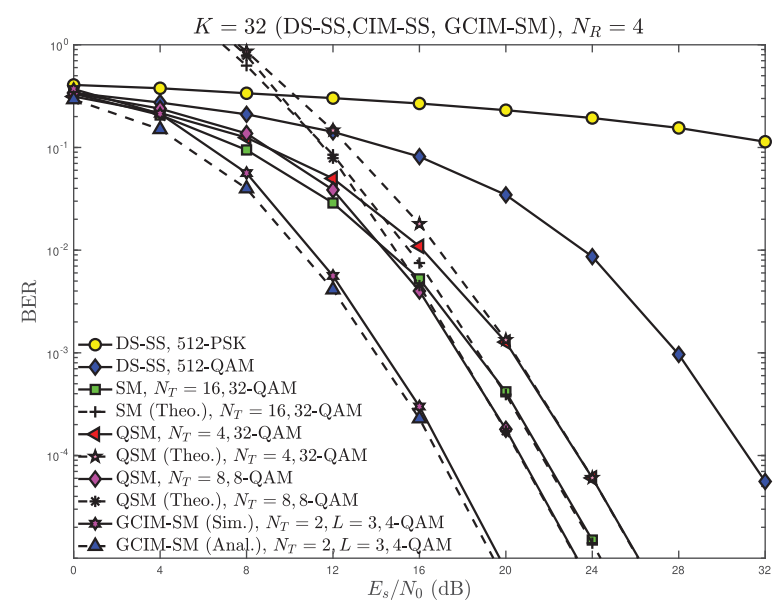

Fig. 3. Performance comparisons of GCIM-SM, CIM-SS, QSM, SM, and DS-SS systems for $p=9 \mathrm{~b}$ when $N_{R}=4$.

and 3, the GCIM-SM technique transmits $9 \mathrm{~b}$ by $1 \mathrm{~b}$ with antenna index, $6 \mathrm{~b}$ with spreading code index, $2 \mathrm{~b}$ with 4-QAM symbol through $I$ and $Q$ components. The SM scheme carries $9 \mathrm{~b}: 4 \mathrm{~b}$ with antenna indices and $5 \mathrm{~b}$ with a 32-QAM symbol. The QSM technique carries $9 \mathrm{~b}$ : In the first scenario, $4 \mathrm{~b}$ with antenna indices and $5 \mathrm{~b}$ with a $32-\mathrm{QAM}$ symbol, in the second scenario, $6 \mathrm{~b}$ with antenna indices and $3 \mathrm{~b}$ with an 8-QAM symbol. In the CIM-SS technique, $6 \mathrm{~b}$ are transmitted in spreading code indices and $3 \mathrm{~b}$ are transmitted with 8-QAM modulation. In the DS-SS technique, all $9 \mathrm{~b}$ are carried on a 512-PSK/QAM modulated symbol. For BER results given in Fig. 2, SNR differences between GCIM-SM and CIM-SS with 8-QAM, DS-SS with 512-QAM, SM, DS-SS with 512-PSK, and QSM with 32 -QAM techniques at $9 \mathrm{~b}$ are $2.7,10,17.7,25$, and $30 \mathrm{~dB}$, respectively. Note that, CIM-SS with 8-PSK and 8-QAM have almost similar BER performance curves. The reason for this can be explained by the fact that the number of antennas in the receiver is insufficient $\left(N_{R}=1\right)$. In other respects, when Fig. 3. is considered, it can straightforwardly be seen that the GCIM-SM system provides 3.6, 4.7, 6.5, and $14.6 \mathrm{~dB}$ SNR gains, respectively, against QSM with $N_{T}=8$ and $M=8 \mathrm{SM}$,

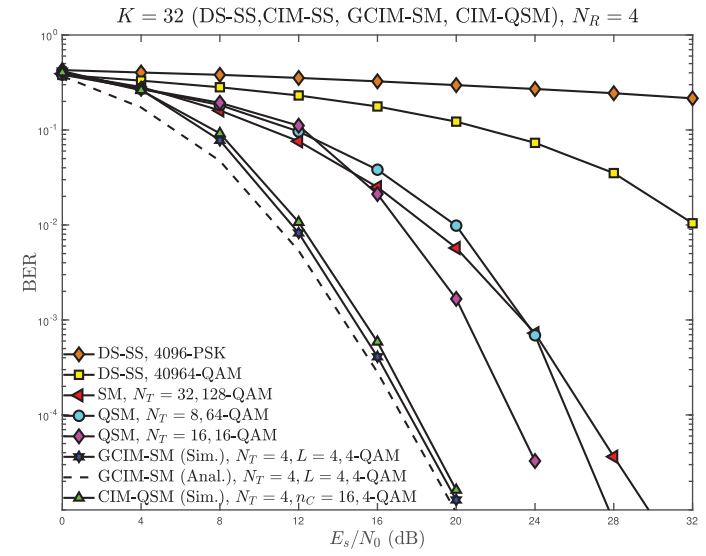

Fig. 4. Performance comparisons of GCIM-SM, CIM-QSM, CIM-SS, QSM, SM, and DS-SS systems for $p=12 \mathrm{~b}$.

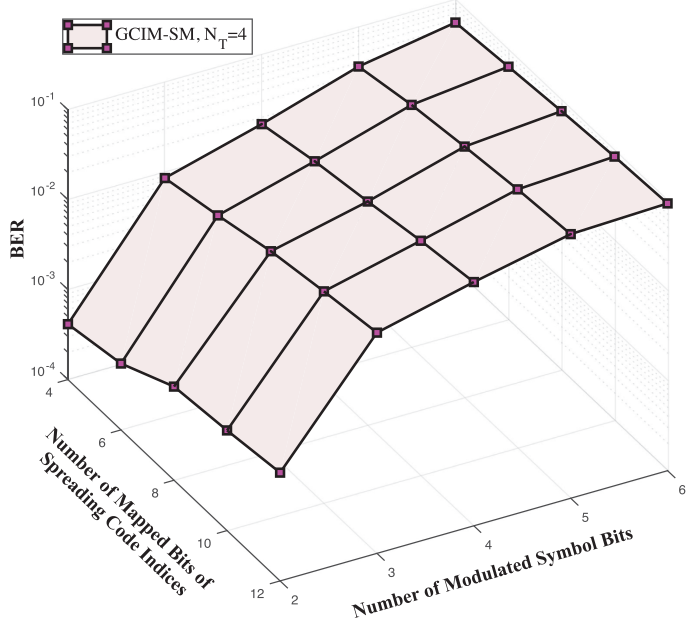

Fig. 5. BER performance curves of the GCIM-SM technique when $N_{T}=4$ and SNR equals $15 \mathrm{~dB}$.

QSM with $N_{T}=4$ and $M=32$, and DS-SS with 512-QAM techniques.

In Fig. 4, BER performance curves of GCIM-SM, CIM-QSM, QSM, SM, and DS-SS for $p=12 \mathrm{~b}$ are presented when $N_{R}=4$. In this figure, $n_{C}$ is the number of spreading codes for CIMQSM. From Fig. 4, it can be seen that the GCIM-SM system provides $5,7.7$, and $9.7 \mathrm{~dB}$ SNR gains, respectively, against QSM with $N_{T}=16$ and $M=16$, SM, QSM with $N_{T}=8$ and $M=64$ techniques.

In Fig. 5, the average BER performance of the GCIM-SM system against number of mapped bits of spreading code indices $(2 L=4,6,8,10,12)$ and number of modulated symbol bits $\left(p_{2}=2,3,4,5,6\right)$ for $\mathrm{SNR}=15 \mathrm{~dB}$ and $N_{T}=4$ is depicted. In a similar manner, the average BER performance of the GCIM-SM system against number of mapped bits of spreading code indices $(2 L=4,6,8,10,12)$ and number of active antenna indices bits $\left(p_{1}=2,3,4,5,6\right)$ for $\mathrm{SNR}=15 \mathrm{~dB}$ and $N_{T}=4$ is depicted. When the average BER performance curves in Figs. 5 and 6 are considered, different data-rate performances are presented where the number of transmitted bits of the GCIM-SM system varies from $p=8$ b to $p=20$ b. Considering Figs. 5 and 6 , it is seen that the performance of the system has deteriorated as a result of the increase of $L$ and $M$. However, for the same 


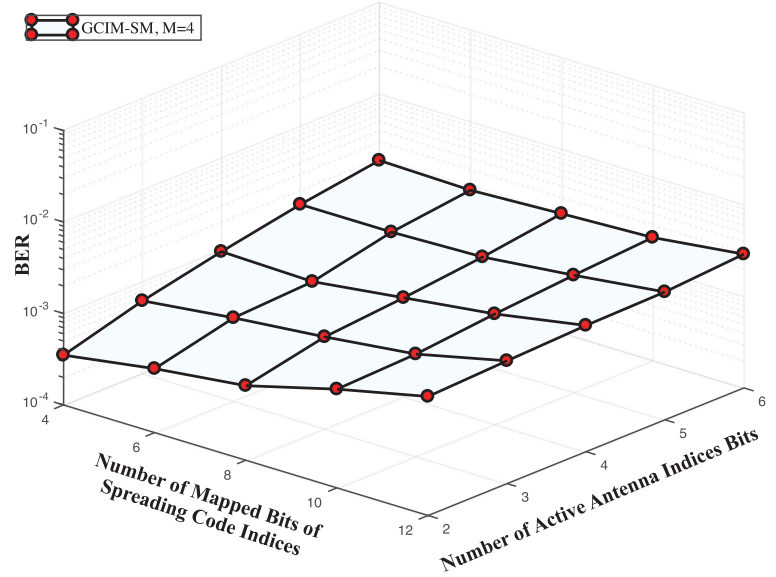

Fig. 6. BER performance curves of the GCIM-SM technique when $M=4$ and SNR equals $15 \mathrm{~dB}$.

$p$-value, the deterioration of performance in Fig. 6 is observed to be less than in Fig. 5 because the active antenna bit is more distorted than the modulated symbol bits.

Consequently, it can be seen from Figs. 2 to 4 that the GCIMSM technique has a significant amount of SNR gain for the same number of bits per symbol and when $N_{R}=1$ and $N_{R}=4$ compared to the above-mentioned techniques.

\section{CONCLUSION}

In this article, the GCIM-SM system, which has a high spectrum and energy efficiency, high data-rate, and better error performance compared to the popular DS-SS, CIM-SS, SM, and QSM techniques, has been introduced. The GCIM-SM system is obtained by combining the CIM and SM techniques and is a generalized form of the system we proposed in [23]. In the GCIM-SM system, not only SMs active antenna indices carry information in the spatial domain but also the spreading code indices. Thus, high data-rate, high spectrum, and energy efficiency have been achieved with very little hardware costs. Our computer simulations show that the GCIM-SM system performs data transmission at higher data-rates, consumes less transmission energy, and performs better than DS-SS, CIM-SS, SM, and QSM techniques.

\section{REFERENCES}

[1] E. Basar, "Index modulation techniques for $5 \mathrm{G}$ wireless networks," IEEE Commun. Mag., vol. 54, no. 7, pp. 168-175, Jul. 2016.

[2] P. Gandotra and R. K. Jha, "A survey on green communication and security challenges in 5G wireless communication networks," J. Netw. Comput. Appl., vol. 96, pp. 39-61, Oct. 2017.

[3] S. H. Alsamhi, O. Ma, M. S. Ansari, and Q. Meng, "Greening Internet of things for smart everythings with a green-environment life: A survey and future prospects," May 2018, arXiv:1805.00844.

[4] N. A. Malik and M. Ur Rehman, "Green communications: Techniques and challenges," EAI Endorsed Trans. Energy Web, vol. 4, Oct. 2017, Art. no. 153162.

[5] A. Younis and R. Mesleh, "Information-theoretic treatment of space modulation MIMO systems," IEEE Trans. Veh. Technol., vol. 67, no. 8, pp. 6960-6969, Aug. 2018.

[6] G. J. Foschini and M. J. Gans, "On limits of wireless communications in a fading environment when using multiple antennas," Wireless Pers. Commun., vol. 6, pp. 311-335, Mar. 1998.
[7] M. Di Renzo, H. Haas, A. Ghrayeb, S. Sugiura, and L. Hanzo, "Spatial modulation for generalized MIMO: Challenges, opportunities, and implementation," Proc. IEEE, vol. 102, pp. 56-103, Jan. 2014.

[8] T. V. H. Nguyen, S. Sugiura, and K. Lee, "Low-complexity sphere searchbased adaptive spatial modulation," IEEE Trans. Veh. Technol., vol. 67, no. 8, pp. 7836-7840, Aug. 2018.

[9] A. E. Canbilen, M. M. Alsmadi, E. Basar, S. S. Ikki, S. S. Gultekin, and I. Develi, "Spatial modulation in the presence of I/Q imbalance: Optimal detector \& performance analysis," IEEE Commun. Lett., vol. 67, no. 8, pp. 7836-7840, Aug. 2018.

[10] J. Y. Wang, J. X. Zhu, S. H. Lin, and J. B. Wang, "Adaptive spatial modulation based visible light communications: SER analysis and optimization," IEEE Photon. J., vol. 10, no. 3, pp. 1-14, Jun. 2018.

[11] M. Arafa, M. Elwekeil, and M. Dessoky, "Mid-symbol duration antenna transition approach for performance enhancement of spatial modulation," Electron. Lett., vol. 54, pp. 506-507, Apr. 2018.

[12] M. Elsayed, H. S. Hussein, and U. S. Mohamed, "Fully generalised spatial modulation," in Proc. 35th Nat. Radio Sci. Conf., Mar. 2018, pp. 274-282.

[13] I. Lai et al., "Spatial permutation modulation for multiple-input multipleoutput (MIMO) systems," IEEE Access, vol. 7, pp. 68206-68218, 2019.

[14] S. Gadhai, A. K. Sah, R. Budhiraja, and A. K. Chaturvedi, "Generalized block-based spatial modulation and space shift keying," in Proc. IEEE 20th Int. Workshop Signal Process. Adv. Wireless Commun., Jul. 2019, pp. 1-5.

[15] E. Aydin and H. Ilhan, "A novel SM-based MIMO system with index modulation," IEEE Commun. Lett., vol. 20, no. 2, pp. 244-247, Feb. 2016.

[16] E. Basar, M. Wen, R. Mesleh, M. Di Renzo, Y. Xiao, and H. Haas, "Index modulation techniques for next-generation wireless networks," IEEE Access, vol. 5, pp. 16693-16746, 2017.

[17] X. Cheng, M. Zhang, M. Wen, and L. Yang, "Index modulation for 5G: Striving to do more with less," IEEE Wireless Commun., vol. 25, no. 2, pp. 126-132, Apr. 2018.

[18] S. Sheng, W. Yang, and Y. Hou, "An improved power spectrum reprocessing method for DS-SS signal on spreading code period estimation," in Proc. 2nd IEEE Adv. Inf. Manage., Communicates, Electron., Autom. Control Conf., May 2018, pp. 774-779.

[19] S. Shiqiang, Y. Wenge, and S. Zeyin, "A blind synchronization method for long code DS-SS signal," in Proc. 3rd IEEE Int. Conf. Comput. Commun., Dec. 2017, pp. 1391-1395.

[20] G. Kaddoum, M. F. Ahmed, and Y. Nijsure, "Code index modulation: A high data rate and energy efficient communication system," IEEE Commun. Lett., vol. 19, no. 2, pp. 175-178, Feb. 2015.

[21] G. Kaddoum and E. Soujeri, "On the comparison between code-index modulation and spatial modulation techniques," in Proc. Int. Conf. Inf. Commun. Technol. Res., May 2015, pp. 1-4.

[22] G. Kaddoum, Y. Nijsure, and H. Tran, "Generalized code index modulation technique for high-data-rate communication systems," IEEE Trans. Veh. Technol., vol. 65, no. 5, pp. 7000-7009, Sep. 2016.

[23] F. Cogen, E. Aydin, N. Kabaoğlu, E. Basar, and H. Ilhan, "A novel MIMO scheme based on code-index modulation and spatial modulation," in Proc. 26th Signal Process. Commun. Appl. Conf., May 2018, pp. 1-4.

[24] F. Cogen, E. Aydin, N. Kabaoglu, E. Basar, and H. Ilhan, "Code index modulation and spatial modulation: A new high rate and energy efficient scheme for MIMO systems," in Proc. 41st Int. Conf. Telecommun. Signal Process., Jul. 2018, pp. 1-4.

[25] E. Aydin, F. Cogen, and E. Basar, "Code-index modulation aided quadrature spatial modulation for high-rate MIMO systems," IEEE Trans. Veh. Technol., vol. 68, no. 10, pp. 10257-10261, Oct. 2019.

[26] M. Au, G. Kaddoum, M. S. Alam, E. Basar, and F. Gagnon, "Joint code-frequency index modulation for IOT and multi-user communications," IEEE J. Sel. Topics Signal Process., vol. 13, no. 6, pp. 1223-1236, Oct. 2019.

[27] F. J. Escribano, A. Wagemakers, G. Kaddoum, and J. V. Evangelista, "Design and performance analysis of an index time-frequency modulation scheme for optical communications," IEEE J. Sel. Topics Signal Process., vol. 13, no. 6, pp. 1403-1416, Oct. 2019.

[28] J. G. Proakis, Digital Communications, 5th ed. New York, NY, USA: McGraw-Hill, 2008.

[29] J. Jeganathan, A. Ghrayeb, and L. Szczecinski, "Spatial modulation: Optimal detection and performance analysis," IEEE Commun. Lett., vol. 12, no. 8, pp. 545-547, Aug. 2008.

[30] D. Tse and P. Viswanath, Fundamentals of Wireless Communication. Cambridge, U.K.: Cambridge Univ. Press, 2004. 